\title{
To resect or not to resect, better yet, who to resect? That is the question after polypectomy of malignant polyps
}

\author{
B. Widmann ${ }^{1} \cdot$ T. Steffen ${ }^{1} \cdot$ Ignazio Tarantino $^{1}$ \\ Received: 4 September 2018 / Accepted: 6 September 2018 / Published online: 26 September 2018 \\ (C) Springer-Verlag GmbH Germany, part of Springer Nature 2018
}

Over the past decades, surgeons had to learn that more extensive surgery is not necessarily associated with a better oncological outcome, especially with modern multimodal treatment approaches. The next logical step in this evolution is to question if under certain circumstances no surgery at all, the so called watch-and-wait strategy, might lead to similar oncological results. In this context, we read with great interest the article titled "Malignant colorectal polyps: Endoscopic polypectomy and watchful waiting is not inferior to subsequent bowel resection. A nationwide propensity score-based analysis" [1]. Including patients from the Danish Colorectal Cancer Group database, the nationwide retrospective propensity score matched study investigated the oncological outcome after watchful waiting compared to subsequent bowel resection after endoscopic removal of malignant polyps. The authors conclude from their research that endoscopic polypectomy and watchful waiting may not be inferior to subsequent bowel resection in regard to overall and disease-free survival. Such a strong conclusion must be based on robust data, which in our opinion is not presented by the authors. The strength of the study is definitely its large population-based sample size and the $100 \%$ follow-up. Yet due to the relevant amount of unknown or missing data and the non-randomized nature of the study, the conclusion drawn by the authors has to be viewed with caution for several reasons.

The first concern arises from an inconsistency regarding oncological treatment principles and missing important data. Oncological colorectal surgery is based on two essential pillars. The first pillar is the complete resection of the primary tumor (R0). The second pillar is the systematic dissection of the lymph nodes which drains the tumor region. If tumor cells remain either on the primary tumor site or in the locoregional lymph

Ignazio Tarantino

ignazio.tarantino@kssg.ch

1 Department of General, Visceral, Endocrine and Transplantation Surgery, Kantonsspital St Gallen, St Gallen, Switzerland nodes, a local recurrence has to be expected. In early-stage tumors like malignant colorectal polyps, a complete endoscopic resection is likely to cure the patient even without surgical resection, given that there are no lymph node metastases. In this case, the patient might be spared the risk of surgical morbidity and mortality without relevant risk of cancer recurrence.

The best way to evaluate whether the primary tumor is completely resected is by pathological assessment. After polypectomy with a tumor-free resection margin of $>1 \mathrm{~mm}$, the relapse risk is $0-2 \%$. A positive resection margin or a tumor-free resection margin of $\leq 1 \mathrm{~mm}$ increases the relapse risk to $21 \%$ [2]. In the current study, only a surprising $64.4 \%$ of patients in the watchful waiting group had a negative resection margin. Meanwhile, $14.2 \%$ had a positive and $21.5 \%$ an uncertain resection margin. In these patients, a re-resection would have been expected. Other factors might have influenced the selection for the watchful waiting approach (e.g., patient's wish, comorbidities, favorable tumor features). Similarly surprising is the high rate of piecemeal resection ( $21.7 \%$ in the watchful waiting group), which does not allow a reliable assessment of the resection margin. A certain selection bias has to be suspected, especially since there was no difference in local recurrence between patients with $\mathrm{R} 1$ and R0 margins ( $2.3 \%$ versus $1.5 \%, p=0.566)$.

The main challenge in selecting patients for a watchful waiting strategy is the risk for lymph node metastases. Patients with high risk of lymph node metastases should undergo oncological resection. This increases the chance for cure and allows correct staging, which is the basis for an adjuvant treatment decision. Patients with a low risk of lymph node metastases might qualify for watchful waiting. There are several known factors that help in estimating the risk of nodal involvement in early-stage colorectal cancer. Bosch et al. was able to identify several risk factors for lymph node metastases in a meta-analysis of 17 studies including 3621 patients. They identified lymphatic invasion (relative risk [RR] 5.2, 95\% confidence interval [CI] 4.0-6.8), submucosal invasion $\geq$ $1 \mathrm{~mm}$ (RR 5.2, 95\%CI 1.8-15.4), tumor budding (RR 5.1, 
95\% CI 3.6-7.3), and poor histological differentiation (RR 4.8, 95\% CI 3.3-6.9) as significant risk factors [3]. Additionally, the depth of infiltration such as the Haggitt level in pedunculated polyps and the Kikuchi level can be helpful to assess the risk of nodal metastases. Polyps with a Haggitt level of 1-3 have a low risk for nodal involvement of $<1 \%$, whereas Haggit level 4 polyps have up to $27 \%$ risk for lymph node metastases $[4,5]$. The Kikuchi level shows a rate for lymph node metastases in $3.4 \%$ in sm1, $8.5 \%$ in sm2, and $22.6 \%$ in sm3 [3]. Clinicians likely combined several factors which they based their treatment decision upon. Hase et al. evaluated five risk factors for lymph node metastases in minimal invasive colorectal cancer. They were able to show that the more risk factors present, the higher the risk for nodal involvement. No lymph node metastases were found in patients with $0-3$ risk factors while 4 factors had a nodal involvement in $33.3 \%$. If all five factors were present, the rate increased to $66.7 \%$ [6]. All these factors were evaluated by the authors as well, but data on these factors were missing in 61.6-96.1\%. This rather large proportion of missing data concerning important factors leaves a high risk for selection bias. It is possible that patients with adverse histological features were more likely to undergo subsequent bowel resection. The fact that among the patients who underwent a subsequent bowel resection eight patients had a $\mathrm{T} 2$ and three patients a T3 tumor supports this assumption. The authors used propensity score matching to reduce the risk of a selection bias. Although propensity score matching is a powerful statistical tool, it can only account for known confounders. A known weakness of this statistical method is its inability to account for unknown parameters. To what extent the unknown parameters might have influenced treatment decision remains unclear. Another disadvantage of propensity score matching is the exclusion of a relevant amount of patients from the analysis. In the current study, from the 692 included patients only 304 were available after matching. This leads to a loss of statistical power. In the unmatched analysis, subsequent bowel resection was significantly superior concerning local recurrence and 5-year overall and disease-free survival. After matching, no significant difference was shown. Nevertheless, subsequent bowel resection was borderline significantly superior regarding local recurrence $(p=0.052)$. A non-significant trend can also be observed in improved 5-year survival rates. The loss of significance might just reflect the loss of power caused by the exclusion of a relevant number of patients.

Secondly, the study engages in little discussion about optimal patient selection for the watchful waiting approach. The author concludes that surgical resection might be avoided because $82.5 \%$ of the subsequent bowel resection group had no residual tumor. But this is only half of the truth, since nodal metastases were identified in $10.5 \%$ of the patients. In other words, 1 out of 10 patients was found with metastatic lymph nodes. It is a matter of perspective- is the glass half full or half empty? In a 10-year period after surgical resection, almost all patients with a stage 1 disease can be cured as shown in a study reporting a relative survival of $96.2 \%$. The survival did not differ significantly from the general population after matching for gender, age, and calendar year [7]. Leaving metastatic lymph nodes behind will inevitably lead to recurrence and adversely impact the excellent oncological prognosis proven after radical resection. In our opinion, the more important question is how to identify patients who would benefit from subsequent resection. Unfortunately, current imaging diagnostics including endosonography, computed tomography (CT), positron emission tomography (PET), or magnetic resonance tomography (MRT) do not offer a reliable assessment of the nodal status. A recent study evaluated the accuracy of MRT and CT in colorectal cancer. The sensitivity and specificity were $42.6 \%$ and $74.1 \%$ for MRT compared to $25.0 \%$ and $41.3 \%$ for CT [8]. Most imaging modalities rely on the size of the lymph nodes to identify potential lymph node metastases. Wang et al. analyzed 128 mesorectal lymph node metastases. In this study, $52 \%$ of the lymph nodes between 5 and $10 \mathrm{~mm}$ were tumor infiltrated [9], highlighting that even normal-sized lymph nodes might be infiltrated by tumor cells. Although imperfect, histopathological factors are especially helpful in assessing the risk for nodal involvement in early colorectal cancer. A reliable histopathologic evaluation is dependent on an en bloc endoscopic resection specimen and a thorough pathological workup. It is essential to reduce the percentage of uncertain/missing information on tumor grade, resection margin, lymphovascular invasion, tumor budding, and infiltration depth. With this information available, clinicians would have reliable and evidence-based criteria to select patients for watchful waiting and spare them a surgical resection. Both the gastroenterologist performing the polypectomy and the pathologist assessing the specimen define how many risk factors can be evaluated. Piecemeal resections in suspected malignant polyps should be strictly avoided.

Last but not least, the watchful waiting approach includes an additional challenge. One question is how the follow-up should be organized. Short interval CT scans and colonoscopies go hand in hand with a potentially harmful radiation dose and possible intervention-related morbidity. Furthermore, due to the incomplete staging, some degree of uncertainty remains. A one in ten chance of tumor recurrence can cause a relevant psychological burden for the patient.

In summary, the high rate of uncertain or missing values leads to an unquantifiable risk for unequal comparison groups and selection bias. Therefore, the results of the study have to be considered carefully. The crucial question is which patients qualify for a watchful waiting strategy, since there is a low risk of residual bowel wall tumor and lymph node metastases. Increasing the en bloc polypectomy rate and more detailed histopathologic evaluation of the known risk factors are the first steps in improving the selection process. The psychological impact of the watch-and-wait strategy must also be 
considered. An early involvement of the patient with detailed information on both the risk of recurrence and the perioperative risk is important. We have to keep in mind, that while some patients might be physically unfit for surgery, others might be psychologically unfit for watch-and-wait.

\section{References}

1. Levic K, Bulut O, Hansen TP, Gögenur I, Bisgaard T (2018) Malignant colorectal polyps: endoscopic polypectomy and watchful waiting is not inferior to subsequent bowel resection. A nationwide propensity scorebased analysis. Langenbecks Arch Surg. https://doi. org/10.1007/s00423-018-1706-x

2. Cooper HS, Deppisch LM, Gourley WK, Kahn EI, Lev R, Manley PN, Pascal RR, Qizilbash AH, Rickert RR, Silverman JF, Wirman JA (1995) Endoscopically removed malignant colorectal polyps: clinicopathologic correlations. Gastroenterology 108(6):1657-1665

3. Bosch SL, Teerenstra S, de Wilt JH, Cunningham C, Nagtegaal ID (2013) Predicting lymph node metastasis in pT1 colorectal cancer: a systematic review of risk factors providing rationale for therapy decisions. Endoscopy 45(10):827-834. https://doi.org/10.1055/s-00331344238
4. Haggitt RC, Glotzbach RE, Soffer EE, Wruble LD (1985) Prognostic factors in colorectal carcinomas arising in adenomas: implications for lesions removed by endoscopic polypectomy. Gastroenterology 89(2):328-336

5. Nivatvongs $\mathrm{S}$, Rojanasakul A, Reiman HM, Dozois RR, Wolff BG, Pemberton JH, Beart RW Jr, Jacques LF (1991) The risk of lymph node metastasis in colorectal polyps with invasive adenocarcinoma. Dis Colon Rectum 34(4):323-328

6. Hase K, Shatney CH, Mochizuki H, Johnson DL, Tamakuma S, Vierra M, Trollope M (1995) Long-term results of curative resection of "minimally invasive" colorectal cancer. Dis Colon Rectum 38(1): $19-26$

7. Tarantino I, Achermann P, Guller U, Ulrich A, Schmied BM, Horber D, Cerny T, Stanga Z, Warschkow R (2013) Relative survival is an adequate estimate of cancer-specific survival: baseline mortalityadjusted 10-year survival of 771 rectal cancer patients. Ann Surg Oncol 20(12):3877-3884. https://doi.org/10.1245/s10434-0133173-5

8. Zhou L, Wang JZ, Wang JT, Wu YJ, Chen H, Wang WB, Cao F, Cheng GX (2017) Correlation analysis of MR/CT on colorectal cancer lymph node metastasis characteristics and prognosis. Eur Rev Med Pharmacol Sci 21(6):1219-1225

9. Wang C, Zhou Z, Wang Z, Zheng Y, Zhao G, Yu Y, Cheng Z, Chen D, Liu W (2005) Patterns of neoplastic foci and lymph node micrometastasis within the mesorectum. Langenbeck's Arch Surg 390(4):312-318. https://doi.org/10.1007/s00423-005-0562-7 